\title{
Biological characteristics and anti-biofilm activity of a lytic phage against vancomycin-resistant Enterococcus faecium
}

\author{
Forough Goodarzi ${ }^{1}$, Masoumeh Hallajzadeh ${ }^{1}$, Mohammad Sholeh ${ }^{1}$, Malihe Talebi ${ }^{1}$, Vahid Pirhajati \\ Mahabadi $^{2,3}$, Nour Amirmozafari ${ }^{1 *}$ \\ ${ }^{I}$ Department of Microbiology, School of Medicine, Iran University of Medical Sciences, Tehran, Iran \\ ${ }^{2}$ Neuroscience Research Center, Iran University of Medical Sciences, Tehran, Iran \\ ${ }^{3}$ Cellular and Molecular Research Center, Iran University of Medical Sciences, Tehran, Iran
}

Received: February 2021, Accepted: August 2021

\begin{abstract}
Background and Objectives: An important leading cause of the emergence of vancomycin-resistant enterococci, especially Enterococcus faecium, is the inefficiency of antibiotics in the elimination of drug-resistant pathogens. Consequently, the need for alternative treatments is more necessary than ever.

Materials and Methods: A highly effective bacteriophage against vancomycin-resistant $E$. faecium called vB-EfmS-S2 was isolated from hospital sewage. The biological properties of phage S2 and its effect on biofilm structures were determined.

Results: Phage S2 was specifically capable of lysing a wide range of clinical E. faecium isolates. According to Electron microscopy observations, the phage S2 belonged to the Siphoviridea family. Suitable pH spectra for phage survival was 5-11, at which the phage showed $100 \%$ activity. The optimal temperature for phage growth was $30-45^{\circ} \mathrm{C}$, with the highest growth at $37^{\circ} \mathrm{C}$. Based on one-step growth curve results, the latent period of phage S2 was 14 min with a burst size of $200 \mathrm{PFU} / \mathrm{ml}$. The phage S2 was also able to tolerate bile at concentrations of 1 and $2 \%$ and required $\mathrm{Mg}^{2+}$ for an effective infection cycle. Biofilms were significantly inhibited and disrupted in the presence of the phage.

Conclusion: According to the results, phage S2 could potentially be an alternative for the elimination and control of vancomycin-resistant E. faecium biofilm.
\end{abstract}

Keywords: Enterococcus faecium; Vancomycin-resistant Enterococcus faecium; Phage therapy; Antibiotic-resistance; Biofilm

\section{INTRODUCTION}

Enterococci are Gram-positive bacteria that are inherently beneficial for healthy individuals (1). These bacteria establish the microbial balance in humans as commensal microflora and are highly adaptable to severe environmental conditions (2). In case enterococci pass from their natural site into the bloodstream, they become opportunistic pathogens, espe- cially in those with an immune-deficiency syndrome or long-term antibiotic usage (3). These bacteria can cause wound infections, urinary tract infections, endocarditis, and bacteremia in susceptible hosts, some of which have been associated with biofilm production (4). The most important reason for the spread or recurrence of these hospital-acquired infections is antibiotic resistance which has dramatically increased over the past recent years (5). The antibiotic

*Corresponding author: Nour Amirmozafari, Ph.D, Department of Microbiology, School of Medicine, Iran University of Medical Scienc-

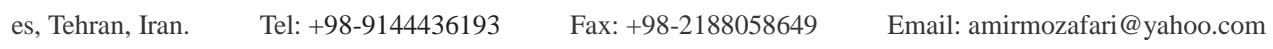


pressure caused by extensive use or misuse of antibiotics can lead to the acquisition of new resistance genes by bacterial strains (6). Among Enterococcus spp., E. faecium which is intrinsically resistant to many common antibiotics, including aminoglycosides, fluoroquinolones, and beta-lactams, has shown high resistance to vancomycin (7). Nowadays, resistance to linezolid and daptomycin has become a challenge during the treatment of vancomycin-resistant E. faecium (8). The mobile genetic elements carrying vancomycin resistance genes can be transferred from resistant $E$. faecium to other opportunistic pathogens, leading to the emergence of resistant species (9). Enterococcus faecium is often considered as the fourth common pathogen leading to hospital-acquired infections worldwide (10). The World Health Organization (WHO) has printed a list of antibiotic-resistant "priority pathogens", with vancomycin-resistant $E$. faecium being in the second place (4). The ability of E. faecium in biofilm formation is also highly important in public health (11). During biofilm formation, microorganisms bind and grow on many surfaces and produce extracellular polymeric substances (EPS) as a matrix (9). The biofilm matrix is highly hydrated (98\% water) and is composed of polysaccharides, proteins, and extracellular DNA (eDNA). The EPS facilitates bacterial attachment and protects the biofilm structure from environmental challenges, such as the immune system and antimicrobial agents (12). The matrix is responsible for the limited penetration of antimicrobial agents into the biofilm (11). A common feature of biofilms is the reduced sensitivity of bacterial cells to antimicrobial compounds, leading to persistent infections. Enterococcal biofilms pose a significant challenge to infection treatment as they are intrinsically resistant to antimicrobial agents (11), the outcome of which is the prolonged infection, increased treatment expenses, and high mortality. Antibiotics have lost their effectiveness in the treatment of resistant enterococcal strains, especially E. faecium; therefore, the elimination of vancomycin-resistant enterococci (VRE) is of great concern (5). Bacteriophages can rapidly inhibit bacterial cells by binding and penetrating them and subsequently disrupting cellular metabolisms (5). The successful use of phages dates back to the 19th century when Felix d'Herelle used them for the treatment of dysentery (5). In phage therapy, the bacteriolytic ability of lytic phage is used to fight and kill multi-drug resistant pathogens, including VRE (13).
Today, one of the most common uses of phages is the management of resistant infections, especially those caused by vancomycin-resistant $E$. faecium (14). The potential advantages of bacteriophages as compared to antibiotics include specific activity, safety, tolerability, easy usage, and site-specific efficiency However, one of the disadvantages of using phage as an antimicrobial agent is the need to study the genome of isolated phage, its high cost and limited range of activity (15). Therefore, phage therapy can potentially be used instead of conventional antibiotics as a promising therapeutic tool. This study aimed to isolate a new phage against vancomycin-resistant $E$. faecium and to determine its physiological characteristics under different conditions. We then demonstrated the efficacy of this phage in the elimination of E. faecium biofilms and the eradication of clinical strains in planktonic forms.

\section{MATERIALS AND METHODS}

Vancomycin-resistant enterococcus isolation. The VRE strains $(n=46)$ were collected from the microbiology laboratory of Shariati hospital (Tehran, Iran). VRE isolates were mainly recovered from urine $(n=30)$, stool $(n=5)$, blood $(n=7)$, catheter $(n=3)$, and B.A.L $(n=2)$ samples of the patients admitted to the Intensive care unit (ICU). Seventeen isolates were from females and 29 isolates were from males. These isolates were identified at the Microbiology Laboratory of Iran University of Medical Science based on conventional microbiological tests (catalase, growth in bile-esculin and BHI agar medium containing $6.5 \%$ salt, PYR assay, motility assay, and arabinose fermentation) (16). For short and long-term storage, isolates were kept in $\mathrm{BHI}$ broth containing $20 \%$ glycerol at $-20^{\circ} \mathrm{C}$ and $-80^{\circ} \mathrm{C}$, respectively.

Antimicrobial susceptibility testing. Antibiotic susceptibility testing was performed by Kirby-Bauer disc diffusion method based on CLSI 2017 guidelines (17). The following antimicrobial disks (Mast Group Ltd., Merseyside, UK.) were used: penicillin (10 units), ciprofloxacin $(5 \mu \mathrm{g})$, teicoplanin $(30 \mu \mathrm{g})$, nitrofurantoin $(300 \mu \mathrm{g})$, linezolid $(30 \mu \mathrm{g})$, and gentamicin $(120 \mu \mathrm{g})$ to detect high-level gentamicin resistance (HLGR) isolates. The minimum inhibitory concentration (MIC) of vancomycin was determined using E-test (ab bio disk, Sweden) strips. Enterococ- 
cus faecalis ATCC29212 and Staphylococcus aureus ATCC25923 were used as controls.

Multiplex polymerase chain reaction. Genomic DNA was extracted as described previously (18). The multiplex PCR assay was conducted to amplify D-alanine-D-alanine ligases specific for E. faecium and vanA genes to confirm the identification of isolates as E. faecium and resistance to vancomycin (18) (Table 1). The PCR program used in this study was as follows: initial denaturation at $94^{\circ} \mathrm{C}$ for $5 \mathrm{~min}, 30$ cycles of denaturation at $94^{\circ} \mathrm{C}$ for $1 \mathrm{~min}$, annealing at $54^{\circ} \mathrm{C}$ for $1 \mathrm{~min}$ and extension at $72^{\circ} \mathrm{C}$ for $1 \mathrm{~min}$ and a final extension at $72^{\circ} \mathrm{C}$ for $10 \mathrm{~min}$.

Isolation of bacteriophages. The isolation of specific bacteriophages was done according to the previously described method with slight modifications (19). In brief, raw sewage samples from Shariati Hospital (Tehran, Iran) were used as a phages source to isolate specific lytic phages of vancomycin-resistant E. faecium. The sewage sample was centrifuged for $15 \mathrm{~min}$ at $4000 \mathrm{rpm}$ to precipitate bacterial cells and debris. The supernatant was passed through a sterile membrane filter $(0.45 \mu \mathrm{m}$ pore size Millipore filters $)$ and stored in clean tube at $4^{\circ} \mathrm{C}$. VREfm 2 (Vancomycin-resistant Enterococcus faecium No. 2) was chosen as a host bacterium and cultured in BHI broth at $37^{\circ} \mathrm{C}$ to reach the logarithmic growth phase. Equal volumes $(10 \mathrm{ml})$ of logarithmic VREfm 2 cultures and sewage filtrates were mixed. Ten milliliters of $2 \times \mathrm{BHI}$ broth medium supplemented with $\mathrm{MgSO}_{4}$ was added and incubated at $37^{\circ} \mathrm{C}$ for $24 \mathrm{~h}$ with shaking at 80 $\mathrm{rpm}$. On the next day, this culture (phage lysate) was centrifuged and examined for phage plaque formation by plaque assay method.

Plaque assay, purification, and titration. The double-layer agar (DLA) method was used for phage plaque assay (20). One $\mathrm{ml}$ of filtrated lysate and $500 \mu \mathrm{l}$ of overnight VREfm2 culture were added to melted BHI soft agar $\left(0.5 \mathrm{mM} \mathrm{MgSO}{ }_{4}, 0.7 \% \mathrm{wt} /\right.$ vol agar, $7 \mathrm{ml}, 45^{\circ} \mathrm{C}$ ). The mixture was spread uniformly on BHI agar plate and incubated at $37^{\circ} \mathrm{C}$ for $24 \mathrm{~h}$. Bacteriophage purification was carried out by three repeated single plaque isolation steps. A single transparent plaque was picked with a sterile Pasteur pipette and transferred to a microtube containing logarithmic-phase culture of VREfm2 in BHI broth. After incubation at $37^{\circ} \mathrm{C}$ for $24 \mathrm{~h}$, the mixture was centrifuged at $10000 \mathrm{rpm}$ for $10 \mathrm{~min}$ and examined for plaque formation by the Double-layer Agar (DLA) method. These steps were repeated thrice to obtain the pure phage. For phage titration, ten sterile microtubes containing $900 \mathrm{ml} \mathrm{SM}$ buffer $(50 \mathrm{mM}$ Tris-HCl, $99 \mathrm{mM} \mathrm{NaCl}, 8 \mathrm{mM} \mathrm{MgSO}$, $0.01 \mathrm{mM}$ gelatin, $\mathrm{pH} 7.5$ ) was used to prepare 10-fold serial dilution. One hundred microliters of phage lysate filtrate was added to the first tube, mixed well and then $100 \mu 1$ of this filtrate was transferred to the second tube. The same pattern was used to make all 10 serial dilutions. Equal volumes $(100 \mu \mathrm{l})$ of the exponential-phase culture of VREfm2 and each dilution of the phage lysate were added to $3 \mathrm{ml}$ of BHI soft agar $\left(45^{\circ} \mathrm{C}\right)$ and poured onto LB agar plates. Plates were incubated at $37^{\circ} \mathrm{C}$ for 18 $-24 \mathrm{~h}$ and the resulting plaques were counted. Phage titration was expressed as plaque-forming units (PFU/ $\mathrm{ml}$ ) as previously described (20). PFU/ml was equal to the number of counted plaques/dilutions $\times$ volume of diluted phages added to each plate.

Electron microscopy. Electron microscopy was used to observe the morphology of the isolated phage particles based on the method described by Hallajzadeh et al. (21). Briefly, $10 \mu \mathrm{l}$ of the purified phage particles was poured on the surface of a carbon-coated copper grid for 3-5 min and then negatively stained by $1 \%$ (w/v) uranyl acetate ( $\mathrm{pH} 7$ ). The grid was air-dried, and images were taken using a Zeiss LEO 906 transmission electron microscope (Carl Zeiss LEO EM $906 \mathrm{E}$, Germany) at an accelerating $100 \mathrm{kV}$ voltage.

Host range determination. The host specificity range for the isolated phages was determined based

Table 1. The Primer sequences used in the multiplex PCR assay

\begin{tabular}{llcc}
\hline Gene & Primer Sequence & Product size & Reference \\
\hline E. faecium & 5'-TTGAGGCAGACCAGATTGACG & 658 & $(18)$ \\
& 5'-TATGACAGCGACTCCGATTCC & & $(18)$ \\
vanA & 5'ATGAATAGAATAAAAGTTGCAATA & 1030 & \\
& 5'-CCCCTTTAACGCTAATACGATCAA & & \\
\hline
\end{tabular}


on Kutter's spot test method (22) with some modifications. Thirty-four clinical vancomycin-resistant $E$. faecium strains were used to determine the host range of isolated phage. Briefly, bacterial strains were cultured in a BHI broth medium at $37^{\circ} \mathrm{C}$ for $24 \mathrm{~h}$. Then, $1 \mathrm{ml}$ of overnight culture of each strain was added to $6 \mathrm{ml}$ of BHI soft agar and spread on BHI agar plate. After drying, $10 \mu \mathrm{l}$ of the filtered phage suspension $\left(1.1 \times 10^{10} \mathrm{PFU} / \mathrm{ml}\right)$ was punctured on the bacterial layer. Following overnight incubation at $37^{\circ} \mathrm{C}$, the plates were checked for bacterial lysis by the phage (plaque formation). The DLA method was also used to confirm the results of spot test. Lytic activity of the isolated phages was also evaluated on vancomycin-resistant E. faecalis, Enterococcus gallinarum, and Escherichia coli.

Temperature stability assay. The effect of temperature on phage viability was assessed according to the method described by Chen et al. (23). The phage suspension $\left(1.1 \times 10^{10} \mathrm{PFU} / \mathrm{ml}\right)$ was inoculated into a microtube containing $\mathrm{BHI}$ broth (at a final volume of $1 \mathrm{~mL}$ ) and incubated at $30,37,45,50$, and $55^{\circ} \mathrm{C}$ for $30 \mathrm{~min}$ at $\mathrm{pH}$ 7. The treated sample was immediately tittered, and phage survival was evaluated by plaque assay. Phage incubated at $37^{\circ} \mathrm{C}$ was considered as control. The effect of temperature on phage survival was assessed by calculating the percentage of active phages versus control.

pH stability assay. The effect of different $\mathrm{pH}$ values on phage viability was assessed as previously described (23). Phage $\left(1.1 \times 10^{10} \mathrm{PFU} / \mathrm{ml}\right)$ was inoculated into a microtube containing BHI broth (at a final volume of $1 \mathrm{~mL}$ ) at $\mathrm{pH} \mathrm{1,} \mathrm{3,} \mathrm{5,} \mathrm{7,} \mathrm{9,} \mathrm{and} 11$ and incubated at $37^{\circ} \mathrm{C}$ for $16 \mathrm{~h}$. The treated sample was immediately tittered as describe above and the DLA assay was used to evaluate phage survival. Phages incubated at $\mathrm{pH} 7$ were used as a control. The effects of different $\mathrm{pH}$ values were assessed based on the percentage of viable phages compared to the control.

Effect of calcium and magnesium ions on phage adsorption rate. Twenty-five milliliter of overnight culture of VREfm2 and the phage lysate was added to four sterile tubes so that the MOI of 0.5 was achieved. $\mathrm{CaCl}_{2}$ or $\mathrm{MgCl}_{2}$ in final concentration of $10 \mathrm{mmol} / \mathrm{L}$ was added in mixtures and incubated at $37^{\circ} \mathrm{C}, 80 \mathrm{rpm}$. Tubes containing the overnight culture of VREfm 2 and the phage lysate without divalent cat- ions were considered as controls. Samples were taken at $0,10,20$, and $30 \mathrm{~min}$ after starting incubation and centrifuged at $14000 \mathrm{rpm}$ for $3 \mathrm{~min}$. The supernatant was titrated for unabsorbed phages using the DLA method. The numbers of free phages were compared to the control. The results were expressed as the proportion of adsorbed phage.

Stability in bile salts (BS). One hundred microliter of the phage suspension $\left(1.1 \times 10^{10} \mathrm{PFU} / \mathrm{ml}\right)$ was added to $9.9 \mathrm{ml} \mathrm{BHI}$ broth containing $1 \%$ and $2 \%$ (wt/ vol) porcine bile extract (Sigma-Aldrich, Oakville, $\mathrm{ON}$, Canada) respectively and incubated for $3 \mathrm{~h}$ at $37^{\circ} \mathrm{C}$. Samples were taken at 0,1 , and $3 \mathrm{~h}$, after starting incubation and immediately, 10-fold serial dilutions were prepared. Phage suspension added to BHI broth without the bile salt was used as a control. The survival rate was reported based on $\log \mathrm{PFU} / \mathrm{ml}$ of treated phage suspension compared to the control.

One-step growth experiment. A one-step growth experiment was conducted as described previously (24). Briefly, $50 \mathrm{ml}$ of mid-exponential phase (OD600 $=0.4-0.6)$ VREfm2 was precipitated by centrifugation (14000 rpm, $5 \mathrm{~min}$ ). The bacterial pellet was suspended in $100 \mu \mathrm{L} \mathrm{BHI}$ broth and mixed with the phage lysate suspension at a MOI of $1 \times 10^{-4}$. This mixture was incubated for $3 \mathrm{~min}$ at room temperature to allow the phage particles to bind the host cell. The mixture was then centrifuged (14000 rpm, 30s) to remove free phages, and the pellet containing adsorbed phages was suspended in $100 \mathrm{ml}$ BHI broth and incubated at $37^{\circ} \mathrm{C}, 120 \mathrm{rpm}$. Samples were taken every $5 \mathrm{~min}$ for $2 \mathrm{~h}$ and after centrifugation (14000 rpm, 1 min), phages were counted by the DLA method. The burst size was calculated as the ratio of the final count of liberated phage particles to the initial count of infected bacteria (infective centers) during the latent period.

Anti-biofilm effect of phage. The anti-biofilm effect of isolated phage was set up in inhibition and disruption experiments as described by Kumar et al. (25) with some modifications. Inhibition experiments were carried out on 1 to 7 -day old biofilms in the presence of the phage. For disruption experiments, 1 to 7-day old biofilms were grown in the presence and absence of the phage. Crystal violet (CV) staining was used to assess the inhibition and disruption effects of the phage. The effect of the phage was com- 
pared with BHI media and 1 to 7-day old biofilms as controls. Biofilm assay was performed in triplicates in both experiments.

Inhibition assay. The VREfm 2 strain was cultured overnight at $37^{\circ} \mathrm{C}$. To prepare log-phase bacterial cells, the overnight culture was sub-cultured in BHI broth medium and incubated for $2 \mathrm{~h}$ with 150 $\mathrm{rpm}$ shaking at $37^{\circ} \mathrm{C}$ to reach the $\mathrm{OD}$ of $\sim 0.5$. Biofilm inhibition assay was carried out by adding $20 \mu \mathrm{l}$ bacterial culture (OD $~ 0.5)$ plus $220 \mu$ l of BHI broth medium and $10 \mu \mathrm{l}$ phage lysate $\left(1.1 \times 10^{10} \mathrm{PFU} / \mathrm{ml}\right)$ in each well of the microtiter plate (Biofil: TCP001096) and incubated for 1 to 7 days at $37^{\circ} \mathrm{C}$. During incubation, until the day of crystal violet (CV) assay, the solution was removed every $24 \mathrm{~h}$, and $240 \mu \mathrm{l}$ fresh BHI medium and $10 \mu \mathrm{l}$ phage suspension were added to each well. After incubation (1 to 7 days), suspension was removed from the wells and washed three times with sterile distilled water to remove planktonic cells. Cells were fixed with $200 \mu$ methanol for 20 min, followed by staining with $200 \mu \mathrm{CV} 1 \%$ (Sigma, C0775) for $20 \mathrm{~min}$. Each well was washed three times with distilled water to remove the extra stain. Then, CV stain in biofilms was dissolved by adding $200 \mu \mathrm{l}$ of ethanol 96\%, and absorption was measured by a plate reader at $630 \mathrm{~nm}$ (Biotech, Synergy2-Cam4, Software-Gen5-1.08). BHI broth containing standard strains of VREfm was used as a positive control. Biofilm assay was performed in triplicates for treated and untreated samples.

Disruption assay. Twenty microliters of VREfm2 (OD 0.5) and $230 \mu \mathrm{l}$ of BHI media were added to each well of 96-well plate and incubated 1 to 7 days at $37^{\circ} \mathrm{C}$. After biofilm establishment within the required duration, treatment with $10 \mu \mathrm{l}$ of phage suspension $\left(1.1 \times 10^{10} \mathrm{PFU} / \mathrm{ml}\right)$ was carried out for $24 \mathrm{~h}$ at $37^{\circ} \mathrm{C}$. The biomass was quantified by absorbance measurements as described before. BHI broth containing standard strains of VREfm was used as a positive control. Biofilm assay was performed in triplicates for treated and untreated samples.

Quantification of the live cells of biofilm by colony-forming unit (CFU) assay. Anti-biofilm efficacy of the phage against the VREfm2 strain was validated as described by Khalifa et al. (6). After incubation of biofilms in both inhibition and degradation assays, planktonic cells were removed and wells were washed with PBS buffer. Biofilm in each well was scraped off using a sterile sharp instrument and suspended in $100 \mu \mathrm{l}$ of PBS buffer. The solutions were prepared in 10-fold serial dilutions and $100 \mu \mathrm{l}$ of each dilution was spread onto BHI plates and incubated at $37^{\circ} \mathrm{C}$ for $24 \mathrm{~h}$.

Statistical analysis. All data were analyzed using GraphPad Prism 6 (GraphPad Software, Inc., San Diego, CA, USA) software. Each experiment was replicated three times. Means were compared with the one-way analysis of variance (ANOVA). Differences with $\mathrm{P}<0.05$ were considered statistically significant. The error bars in the figures represent the standard deviation $(\mathrm{SD})$ of the mean.

\section{RESULTS}

Vancomycin-resistant $\boldsymbol{E}$. faecium isolation. A total of 47 VRE isolates were recovered from patients admitted to Shariati hospital in Tehran, Iran, and identified to the species level. Out of the 47 isolates, 34 were identified as E. faecium. The PCR results confirmed that all E. faecium were vanA-positive. The results of the multiplex PCR are presented in Fig. 1A.

\begin{abstract}
Antimicrobial sensitivity test. Enterococcus faecium isolates exhibited high rates of resistance to ciprofloxacin $(88.2 \%)$, teicoplanin $(67.4 \%)$, and nitrofurantoin $(26.4 \%)$. The highest resistance was against penicillin $(100 \%)$, while the lowest resistance was against linezolid $(2.9 \%)$. HLGR was found in $61.76 \%$ of E. faecium isolates. All E. faecium isolates were highly resistant to vancomycin with a MIC of $\geq 256 \mu \mathrm{g} / \mathrm{ml}$.
\end{abstract}

Isolation of bacteriophages. Four lytic phages were isolated from two different wastewater samples from Shariati hospital using the DLA technique and the VREfm2 was used as the host strain. Among these, one phage was selected for further experiments based on the high phage titer. This phage formed translucent plaques with a diameter of 1-2 mm without any halo in the host strain (Fig. 1B).

Electron microscopy. According to the TEM analysis, the isolated phage had an icosahedral head and a flexible tail with a diameter of $60 \pm 0.3 \mathrm{~nm}$ and 135 $\pm 0.1 \mathrm{~nm}$, respectively. This morphology indicates that the VREfm2-specific phage belongs to the $\mathrm{Si}$ - 
phoviridea family and Caudovirales order according to the ICTV classification system (Fig. 1C). According to the newly proposed naming system, this phage was named vB-EfmS-S2 and is commonly known as phage $S 2$ (26).

Host range determination. The host range of the phage S2 was determined using 34 E. faecium isolates and other clinical strains (Table 2). The results showed that phage $\mathrm{S} 2$ could efficiently lyse 28 E. faecium strains $(82.3 \%)$, reflecting its relatively broad host specificity on recovered E. faecium isolates. However, phage S2 could not infect any of E. faecalis, E. gallinarum, and E. coli isolates.

Thermal stability on phage viability. As illustrated in Fig. 2A, phage S2 maintained its viability at $37^{\circ} \mathrm{C}$ after $30 \mathrm{~min}$ as a control; however, its viability was significantly affected at higher temperatures (50$\left.55^{\circ} \mathrm{C}\right)(\mathrm{p}<0.05)$. After $30 \mathrm{~min}$ of incubation, phage titers showed $40 \%$ and $90 \%$ reduction at 50 and $55^{\circ} \mathrm{C}$, respectively, compared to the control. Phage S2 titer decreased $10 \%$ at 30 and $45^{\circ} \mathrm{C}$. Therefore, this phage has a better survival rate at these temperatures as a slight decrease in titer was observed compared to the control after $30 \mathrm{~min}$ of incubation.

pH stability test on phage viability. After $16 \mathrm{~h}$ incubation in different $\mathrm{pH}$ values at $37^{\circ} \mathrm{C}$, phage $\mathrm{S} 2$ could survive at $\mathrm{pH} 1$ and 3 ; however, phage viability showed $30 \%$ and $20 \%$ reduction, respectively, and small plaques formed when compared to $\mathrm{pH} 7$. Also,
Table 2. Lytic spectrum of phage S2 against a panel of clinical isolates

\begin{tabular}{lcc}
\hline $\begin{array}{l}\text { Bacterial } \\
\text { strain }\end{array}$ & $\begin{array}{c}\text { Number of } \\
\text { isolates }\end{array}$ & $\begin{array}{c}\text { Plaque } \\
\text { formation }\end{array}$ \\
\hline VREfm & 34 & $82.3 \%$ \\
E. gallinarum & 1 & Not susceptible \\
E. faecalis & 13 & Not susceptible \\
E. coli & 6 & Not susceptible \\
\hline
\end{tabular}

at $\mathrm{pH}$, less stability was observed compared to $\mathrm{pH} 7$ (with $15 \%$ reduction in viability). Optimal lytic phage activity (100\%) was shown at pH 7 and pH 9. Overall, there were no significant differences in phage survival in the $\mathrm{pH}$ range of 7-11 ( $\mathrm{P}$-value > 0.05). This phage was also stable at $\mathrm{pH} 11$ and $9 \%$ reduction in phage titer was observed after $16 \mathrm{~h}$ incubation (Fig. 2B).

Effects of calcium and magnesium ions on phage adsorption. The effect of $\mathrm{Ca}^{2+}$ and $\mathrm{Mg}^{2+}$ ions were investigated on phage adsorption by host receptors. As shown in Fig. 2C, cell lysis occurred even without divalent cations. Statistical analysis showed a significant difference in phage adsorption rate in samples treated with $\mathrm{Mg}^{2+}$ when compared to the control (10\% increase in absorption after $30 \mathrm{~min}$ of incubation). The number of free phages decreased significantly compared to the control, indicating the elevated rate of phage uptake by the bacteria (P-value $<0.05)$. On the contrary, $\mathrm{Ca}^{2+}$ showed no significant effect on phage uptake over time.

1A

$1 \mathrm{C}$

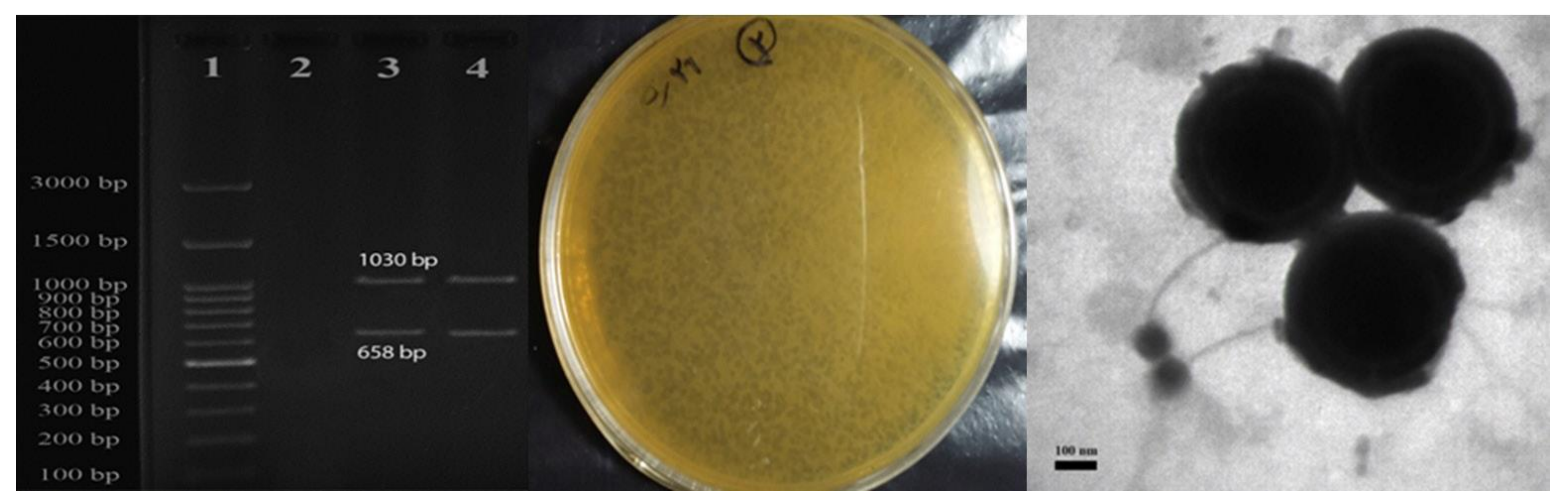

Fig. 1. Electrophoresis of multiplex PCR products of E. faecium-specific and vanA genes on the $0.1 \%$ agarose gel with 100 -bp DNA ladder. Lane 1: 100-bp DNA ladder (SMO Bio), Lane 2: Negative control, Lane 3: positive control, Lane 4: Clinical strain (A). Morphology of phage S2 plaques in the DLA method (B). Transmission electron microscopy (TEM) micrograph of phage S2. The scale bar represents $100 \mathrm{~nm}(\mathrm{C})$. 
Bile salt resistance. Fig.2D shows the relative resistance of phage S2 to 1 and $2 \%$ bile salt. Generally, a significant tolerance of bacteriophage to high bile concentrations was observed. Concentrations of $1 \%$ and $2 \%$ bile salt showed no significant effect on phage growth after $1 \mathrm{~h}(\mathrm{P}$-value > 0.05). However, after $3 \mathrm{~h}$, the stability of phages decreased significantly in both concentrations (P-value <0.05). The results indicated that the concentration of phage $S 2$ decreased $0.47 \log _{10}$ and $0.66 \log _{10}$ in the presence of 1 and $2 \%$ bile, respectively, after $3 \mathrm{~h}$.

One-step growth curve. The one-step growth curve of phage S2 showed that the latent period was 14.86 min, and the burst time was $95 \mathrm{~min}$. The burst size was 200 PFU/cell (Fig. 2E).

Inhibition assay. The absorbance values (OD630 $\mathrm{nm}$ ) of treated biofilms on days 1-3 were almost equal. The bacteriophage inhibited biofilm formation for 1-5 days and exerted a lesser effect on biofilms on days 6 and 7. The absorbance (OD630 nm) values of treated biofilms on days 1-7 were significantly lower than untreated biofilms on days 1-7. Biofilm inhibition was three-fold greater than the control. The logarithmic growth model shows the data regarding treated and untreated biofilms (Fig. 3).

Disruption assay. As shown in Fig. 4, the phage significantly penetrated biofilms on days 1-4 and destroyed its impermeable structure; however, it had a

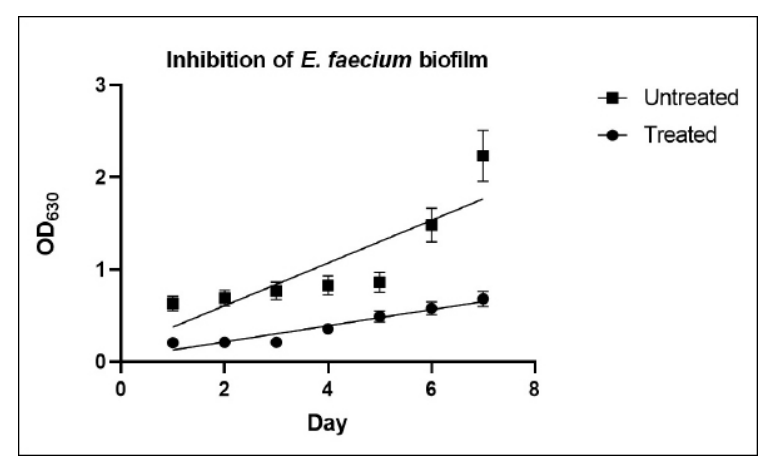

Fig. 3. Inhibitory effect of phage $\mathrm{S} 2$ on VREfm2 biofilm. Nonlinear regression diagram of the assessment of biofilm inhibition. Comparison of biofilm inhibition by phage $\mathrm{S} 2$ at different time intervals (days 1-7) compared to the control at $630 \mathrm{~nm}$.
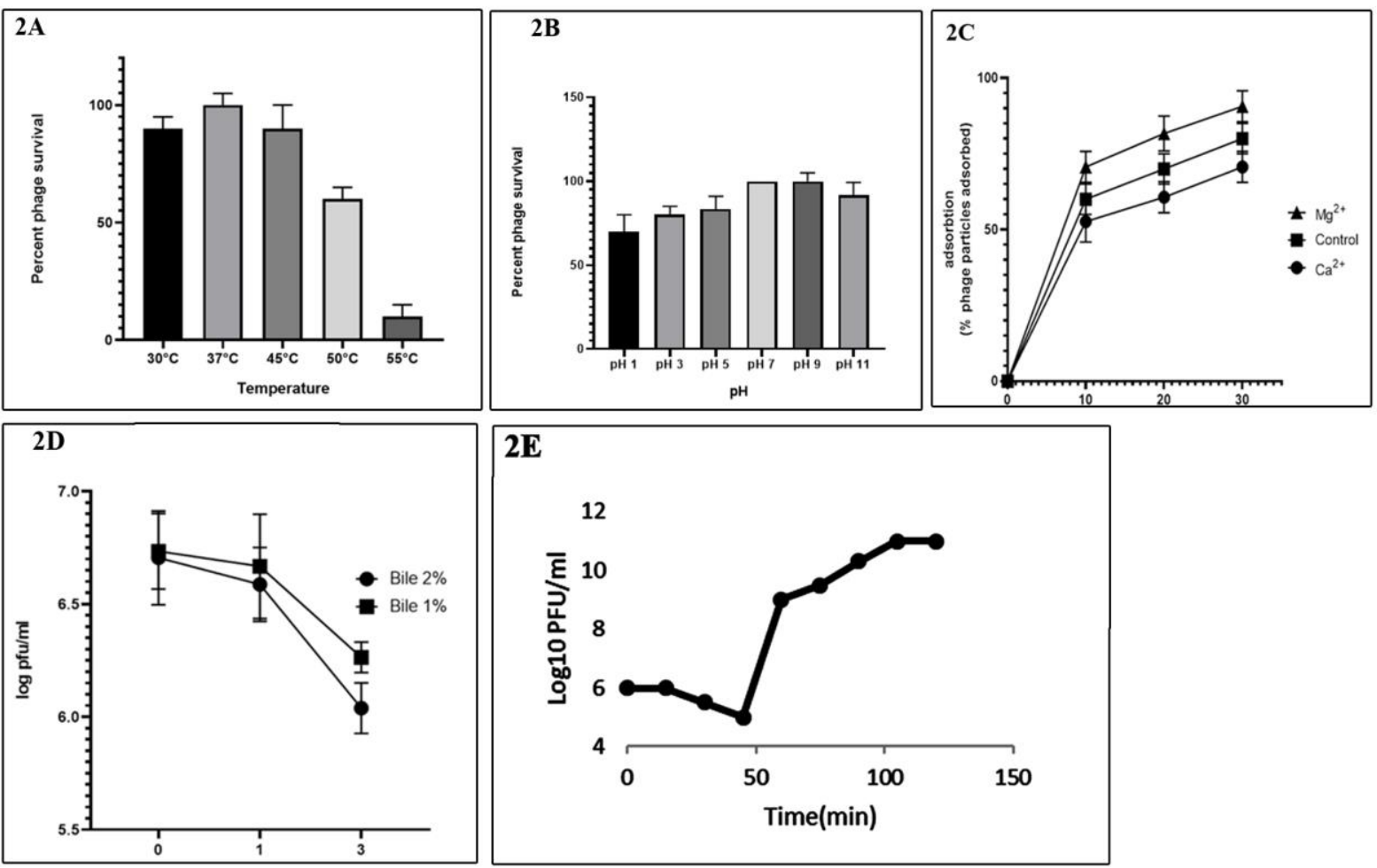

Fig. 2. Survival rate of phage $S 2$ at different temperatures (A). Stability of phage $S 2$ at different pH levels (B). Absorption rate of phage S2 in the presence of magnesium and calcium ions (C). Survival rate of phage S2 after exposure to 1 or $2 \%$ bile salts (D). One-step growth curve of phage S2 (E). 
less significant effect on days 5-7. The phage could significantly degrade treated biofilms after 1-7 days with varying intensity compared to untreated biofilms. Degradation of the treated biofilm was twice higher compared to the control. The logarithmic growth model shows the data regarding treated and untreated biofilms (Fig. 4).

Colony-forming unit assay following inhibition and disruption of the biofilm. As there are both dead and living cells in the biofilm structure after phage treatment, CV staining does not provide information on the number of living cells within the biofilm structure. For this purpose, the CFU assay was performed to enumerate the living cells within the biofilm structure. According to the CFU values, phage $\mathrm{S} 2$ reduced the number of living cells within the biofilm structure for up to 7 days compared to the

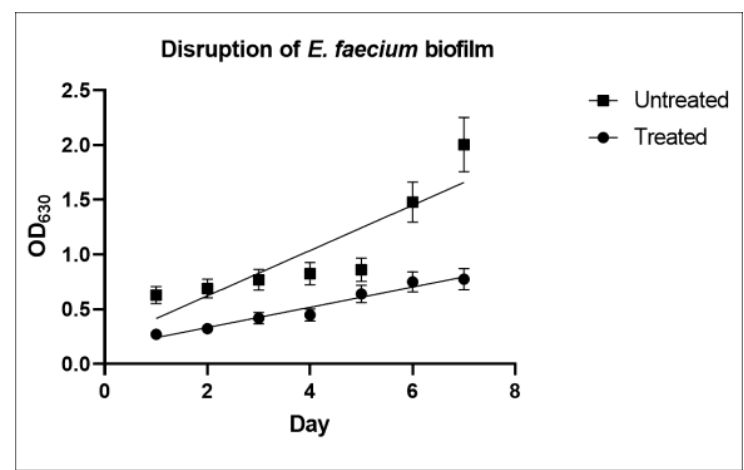

Fig. 4. Disruptive effect of phage $S 2$ on VREfm2 biofilms. Nonlinear regression diagram of biofilm disruption assessments. Biofilm disruption with phage S2 at different time intervals (days 1-7) compared to the control at $630 \mathrm{~nm}$. control by both destruction and inhibition (Fig. 5). This reduction following inhibitory and destructive effects was $2.5 \log$ and $2 \log$, respectively, compared to the control over the course of 7 days.

\section{DISCUSSION}

Antibiotic resistance is now a serious threat to human health around the world (27). Today, the emergence rate of antibiotic resistance has surpassed the efficiency of antibiotics (28). Enterococci have been known as pathogens for more than a hundred years. These bacterial spp. have also emerged as important hospital pathogens in the last few decades and have become a severe health problem ever since (29). Unlike antibiotics that require a long time for design and synthesis, effective phages can be readily isolated following the emergence of new resistance. To this end, the use of phages to suppress resistant bacteria has given clinicians hopes for the future of infection therapy. In this investigation, 34 vanA positive E. faecium isolates were recovered from clinical samples. We isolated four specific lytic phages for VREfm host strains from hospital sewage comparable with our experiment, Vancomycin-resistant $E$. faecium-specific phages have been isolated in other studies $(19,20,24)$. One phage was selected for further evaluations based on a broader host spectrum and larger plaque size. Electron microscopy observation demonstrated that the isolated phage belonged to the Siphoviridea family. Similar to our study Li et al. isolated E. faecium-infecting Ec-ZZ2 phage with characteristics of the Siphoviridea family (30) .De-
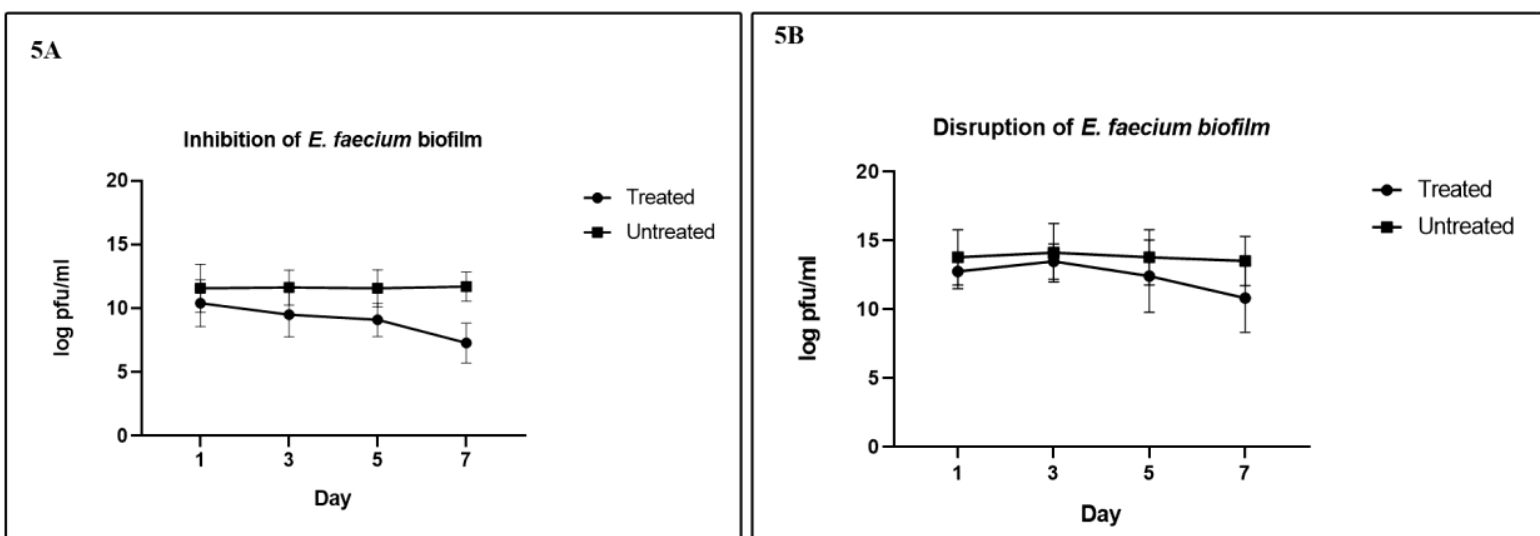

Fig. 5. Colony-forming unit assay for the enumeration of living cells in VREfm2 biofilms. Log10 CFU of live VREfm2 in different days. Biofilm was treated with and without the phage under inhibition (A) and disruption (B) conditions. 
termining the hosting spectrum defines the biological characteristics of the phage and is a crucial step in phage therapy (31). The host range of our isolated phage was examined on 34 clinical VREfm strains by the spot test. The phage $\mathrm{S} 2$ was able to attack 28 (out of 34) clinical strains and showed broad lytic spectrum $(82.3 \%)$ against E. faecium, which makes phage S2 an appropriate candidate for controlling $E$. faecium infections. Physicochemical factors such as temperature, acidic and alkaline $\mathrm{pH}$, bile salt, and divalent cations $\left(\mathrm{Ca}^{2+}, \mathrm{Mg}^{2+}\right)$ are considered as factors affecting phage survival in vitro. Alterations in these factors may cause severe damage to the viral structure (32). The resistance of bacteriophages to external factors can make them useful tools to combat resistant bacteria in agriculture and medicine (33). Temperature is a key factor for phage growth and survival and can potentially affect binding, penetration, and proliferation, as well as designing strategies for the storage of the therapeutic phages. In addition, phages that can survive a wide range of temperatures can reduce the maintenance cost. The optimum temperature for the biological activity of phage S2 was $37^{\circ} \mathrm{C}$; however, the plaque formation decreased with increasing temperature from $37^{\circ} \mathrm{C}$ to $55^{\circ} \mathrm{C}$ but phage $\mathrm{S} 2$ exhibited acceptable stability in this temperature range. These findings are in agreement with previous studies $(24,34)$. In this study, phage stability was investigated in a wide range of $\mathrm{pH}$ values (from 1 to 11). The optimal $\mathrm{pH}$ range for the physical stability of phage $\mathrm{S} 2$ was from 7 to 9 as no decrease in phage titer was observed in this $\mathrm{pH}$ range. Hence, the isolated bacteriophage was highly active and stable in alkaline environments, making it compatible with the alkaline condition of the hospital sewage. Phage $\mathrm{S} 2$ also was able to form plaques in the acidic $\mathrm{pH}$ (1 to 3 ); therefore, it could easily survive in acidic and alkaline conditions in different parts of the gastrointestinal tract when administered orally. Similar to our reports, Chen et al. (2019) showed that the $\mathrm{pH}$ range of 5-9 had no significant effect on the viability of the phage P2 (23).

Divalent ions such as $\mathrm{Ca}^{2+}$ and $\mathrm{Mg}^{2+}$ can affect the binding, uptake, and penetration of phages to host cells. These ions facilitate the host-phage interaction by altering bacterial surface receptors and neutralizing the cell surface charge. In this study, the effect of $\mathrm{Ca}^{2+}$ and $\mathrm{Mg}^{2+}$ on phage uptake rate was assessed. According to the results, phage S2 required $10 \mathrm{mM}$ $\mathrm{Mg}^{2+}$ for maximum infectivity, while the presence of
$\mathrm{Ca}^{2+}$ had no significant effect on phage uptake. Similar to our report, Taskeen Raza et al. (2018) reported that $\mathrm{Mg}^{2+}$ significantly increased phage uptake into the host cell and improved plaque formation (24). In contrast to our study, Sidra Rahmat Ullah et al. reported that the addition of $\mathrm{Ca}^{2+}$ affected phage SRG1 absorption (34). Briggiler Marco et al. (2010) showed that divalent ions were not required for phage adsorption and the lytic cycle (35). We also examined the stability of phage $\mathrm{S} 2$ in bile salts. The isolated phage showed high viability in the presence of 1 to $2 \%$ bile salts, whereas the concentration of bile acid produced by the organisms is approximately $0.7 \%$ (36). These findings suggest that phage S2 is likely to maintain its viability in the intestinal fluids. There are few reports on the tolerance of bacteriophages to bile salts. However, our results are consistent with the study of Nobrega et al in 2016 who demonstrated phage tolerance to 1 and $2 \%$ bile salt after $3 \mathrm{~h}$ of incubation (33). In contrast to our findings, several researchers have reported adverse effects of bile salts on phage stability and survival $(37,38)$. The triphasic curve obtained from one-step growth experiment of phage S2 showed a short latent period of $14 \mathrm{~min}$ and a large burst size of 200 virions per cell. These properties indicate the high therapeutic potential of phage S2 as 14 min is needed to replicate the virus inside the host bacteria and high numbers of new phage particles are released in one life cycle. Khalifa et al. and Zhang et al. reported various burst sizes and latent times, indicating that both latent time and burst size vary among different bacteriophages (6, 39). The main focus of this study was to characterize a lytic phage and assess its effect on the biomass reduction of $E$. faecium biofilm by both inhibition and disruption experiments. As indicated by the $\mathrm{CV}$ assay (Figs. 3 and 4), the effect of phages on biofilm inhibition and degradation was significant in initial days; however, older biofilms exhibited less susceptibility compared to the younger ones. Degradation of extracellular polymeric substances of biofilms by the phage $\mathrm{S} 2$ is probably easier for younger biofilms, because of the high density of bacterial cells and the rapid spread of phages in the biofilm structure (40). In contrast, in older biofilms, the formation of a thicker matrix prevents the phage from accessing inner layers of biofilm (41). However, it should be noted that the mechanism of phage-biofilm interaction is not entirely understood (42).

Additionally, according to our findings, phage S2 
has been able to decrease biofilm biomass for two or three folds when compared with untreated biofilm. Therefore, phage S2 possesses the high potential of being successfully used as a biofilm eradication agent. As shown in Fig. 3, the inhibition assay indicated that the isolated phage significantly reduced biofilm biomass compared to the control. The CFU assay of the inhibition experiment indicated that the isolated phage significantly reduced E. faecium cell numbers (for about $2.5 \log$ CFU.mL ${ }^{-1}$ ) within a week (Fig. 5A). This reduction rate is greater than those reported in other studies. For example, LDR Melo et al. reported that their studied lytic phage [vB_EfaS-Zip (Zip)] against $E$. faecium biofilm caused a reduction in bacterial count about $1.5 \log$ CFU.mL $L^{-1}$ following 3-6 h of treatment (43). The CV assay results showed that the isolated phage was more effective in the eradication of 1 to 4 -day old biofilms (Fig. 4). The complex structure of older biofilms partially blocks phage entry. Also, the reduction of bacterial count for $2 \log$ CFU.mL $L^{-1}$ in the degradation experiment verified the destructive effect of phage on bacterial growth (Fig. 5B). Our work demonstrates that phage S2 was more successful in preventing the onset of biofilm development rather than the elimination of an existing biofilm. Reduced biomass and bacterial population in treated biofilms show that phage therapy can be active against biofilm by degradation of EPS and decreasing the number of viable cells (log CFU.mL ${ }^{-1}$ ). Similar to the present study, several recent reports have shown phage activity against biofilm eradication $(44,45)$.

\section{CONCLUSION}

Vancomycin-resistant enterococci infections pose a major public health problem due to limited treatment options. Thus, given the cost imposed by mortality and morbidity, finding a suitable solution to combat such resistant bacteria is critical, especially following the failure of antibiotics. Specific lytic phages against E. faecium have the potential of becoming new alternatives to antibiotics. As phages possess features such as high stability in a wide range of temperature and $\mathrm{pH}$ as well as other physicochemical conditions, they impose low maintenance costs on the health care system. Also, a short latent time, large burst size, and broad host range as well as activity against bacterial biofilms eradication, confirm their high therapeutic potential to control human infections. VRE-related infections have significantly increased in the last decade with limited treatment options; therefore, finding an alternative to conventional antibiotics is of paramount importance. In this research, we successfully isolated a novel phage and evaluated the effect of physicochemical factors on its viability and adsorption rate. This phage showed a broad host range among E. faecium strains. Additionally, the high stability of the phage in a wide range of temperatures and $\mathrm{pH}$ values, as well as short latent period and large burst size confirm the suitable therapeutic application of this phage in controlling human infections. The presence of phage decreased biofilm integrity and adhesion in both inhibition and degradation experiments. The efficacy of the phage against biofilms suggests its therapeutic use; however, more detailed studies are needed.

\section{ACKNOWLEDGEMENTS}

This study was financially supported by a research grant (No. 97-01-30-33074) for an M.Sc. thesis at Iran University of Medical Sciences (Tehran, Iran) for which we are very grateful.

\section{REFERENCES}

1. Ahmed MO, Baptiste KE. Vancomycin-resistant enterococci: a review of antimicrobial resistance mechanisms and perspectives of human and animal health. Microb Drug Resist 2018;24:590-606.

2. Bin-Asif H, Ali SA (2019). The genus Enterococcus and its associated virulent factors. In: Microorganism. Eds, M Blumenberg, M Shaaban, A Elgaml. IntechOpen, $1^{\text {st }}$ ed, London, pp. 109-130.

3. Uchiyama J, Takemura I, Satoh M, Kato S, Ujihara T, Akechi K, et al. Improved adsorption of an Enterococcus faecalis bacteriophage $\Phi \mathrm{EF} 24 \mathrm{C}$ with a spontaneous point mutation. PLoS One 2011;6(10):e26648.

4. Cui P, Feng L, Zhang L, He J, An T, Fu X, et al. Antimicrobial resistance, virulence genes, and biofilm formation capacity among Enterococcus species from Yaks in Aba Tibetan autonomous prefecture, China. Front Microbiol 2020;11:1250.

5. Khalifa L, Shlezinger M, Beyth S, Houri-Haddad Y, Coppenhagen-Glazer S, Beyth N, et al. Phage therapy against Enterococcus faecalis in dental root canals. $J$ Oral Microbiol 2016;8:32157. 
6. Khalifa L, Gelman D, Shlezinger M, Dessal AL, Coppenhagen-Glazer S, Beyth N, et al. Defeating antibiotic-and phage-resistant Enterococcus faecalis using a phage cocktail in vitro and in a clot model. Front Microbiol 2018;9:326.

7. Castillo-Rojas G, Mazari-Hiríart M, de León SP, Amieva-Fernández RI, Agis-Juárez RA, Huebner J, et al. Comparison of Enterococcus faecium and Enterococcus faecalis strains isolated from water and clinical samples: antimicrobial susceptibility and genetic relationships. PLoS One 2013;8(4):e59491.

8. Paganelli FL, Huebner J, Singh KV, Zhang X, van Schaik W, Wobser D, et al. Genome-wide screening identifies phosphotransferase system permease BepA to be involved in Enterococcus faecium endocarditis and biofilm formation. $J$ Infect Dis 2016;214:189-195.

9. Donlan RM. Biofilm formation: a clinically relevant microbiological process. Clin Infect Dis 2001;33:13871392.

10. Akpaka PE, Kissoon S, Wilson C, Jayaratne P, Smith A, Golding GR. Molecular characterization of vancomycin-resistant Enterococcus faecium isolates from Bermuda. PLoS One 2017;12(3):e0171317.

11. Ch'ng JH, Chong KKL, Lam LN, Wong JJ, Kline KA. Biofilm-associated infection by enterococci. Nat Rev Microbiol 2019;17:82-94.

12. Lewis K. Riddle of biofilm resistance. Antimicrob Agents Chemother 2001;45:999-1007.

13. Harada LK, Silva EC, Campos WF, Del Fiol FS, Vila M, Dąbrowska K, et al. Biotechnological applications of bacteriophages: state of the art. Microbiol Res 2018;212-213:38-58.

14. Shlezinger M, Coppenhagen-Glazer S, Gelman D, Beyth N, Hazan R. Eradication of vancomycin-resistant enterococci by combining phage and vancomycin. Viruses 2019;11:954.

15. Principi N, Silvestri E, Esposito S. Advantages and limitations of bacteriophages for the treatment of bacterial infections. Front Pharmacol 2019;10:513.

16. Sattari-Maraji A, Jabalameli F, Farahani NN, Beigverdi R, Emaneini M. Antimicrobial resistance pattern, virulence determinants and molecular analysis of Enterococcus faecium isolated from children infections in Iran. BMC Microbiol 2019;19:156.

17. Clinical and Laboratory Standards Institute [CLSI] (2017). Performance Standards for Antimicrobial Susceptibility Testing M02-A12 M-A, and M11-A8, 27th Ed, Philadelphia, PA: Clinical and Laboratory Standards Institute.

18. Talebi M, Pourshafiei MR, Oskouei M, Eshraghi SS. Molecular analysis of vanHAX element in vancomycin resistant enterococci isolated from hospitalized patients in Tehran. Iran Biomed J 2008;12:223-228.

19. Gong P, Cheng M, Li X, Jiang H, Yu C, Kahaer N, et al. Characterization of Enterococcus faecium bacteriophage IME-EFm5 and its endolysin LysEFm5. Virology 2016;492:11-20.

20. Wang Y, Wang W, Lv Y, Zheng W, Mi Z, Pei G, et al. Characterization and complete genome sequence analysis of novel bacteriophage IME-EFm1 infecting Enterococcus faecium. J Gen Virol 2014;95:2565-2575.

21. Hallajzadeh M, Mojtahedi A, Amirmozafari N, Pirhajati Mahabadi V. Characterizing a lytic bacteriophage infecting methicillin-resistant Staphylococcus aureus (MRSA) isolated from burn patients. Arch Clin Infect Dis 2020;15(1):e91634.

22. Kutter E (2009). Phage host range and efficiency of plating. In: Bacteriophages, methods and protocols. Eds, MR Clokie, AM Kropinski. Springer, $1^{\text {st }}$ ed. Switzerland AG, pp. 141-149.

23. Chen X, Guo J, Liu Y, Chai S, Ma R, Munguntsetseg B. Characterization and adsorption of a Lactobacillus plantarum virulent phage. J Dairy Sci 2019;102:38793886.

24. Raza T, Andleeb S, Ullah SR, Jamal M, Mehmood K, Ali M. Isolation and characterization of a phage to control vancomycin resistant Enterococcus faecium. Open Life Sci 2018;13:553-560.

25. Kumar L, Cox CR, Sarkar SK. Matrix metalloprotease-1 inhibits and disrupts Enterococcus faecalis biofilms. PLoS One 2019;14(1):e0210218.

26. Adriaenssens EM, Wittmann J, Kuhn JH, Turner D, Sullivan MB, Dutilh BE, et al. Taxonomy of prokaryotic viruses: 2017 update from the ICTV bacterial and archaeal viruses Subcommittee. Arch Virol 2018;163:1125-1129.

27. Golkar Z, Bagasra O, Pace DG. Bacteriophage therapy: a potential solution for the antibiotic resistance crisis. $J$ Infect Dev Ctries 2014;8:129-136.

28. Li B, Webster TJ. Bacteria antibiotic resistance: new challenges and opportunities for implant-associated orthopedic infections. J Orthop Res 2018;36:22-32.

29. Higuita NIA, Huycke MM (2014). Enterococcal disease, epidemiology, and implications for treatment. In: Enterococci: from commensals to leading causes of drug resistant infection. Massachusetts eye and ear, Last updated, Boston, pp. 65-99.

30. Li J, Shi H, Zhao C, Hao Y, He Y, Sun Y. Complete genome sequence of the siphoviral bacteriophage EcZZ2, which is capable of lysing Enterococcus faecium. Genome Announc 2016;4(6):e01167-16.

31. Bibi Z, Abbas Z, Rehman SU. The phage P. E1 isolated from hospital sewage reduces the growth of Escherichia coli. Biocontrol Sci Technol 2016;26:181-188.

32. Jończyk E, Kłak M, Międzybrodzki R, Górski A. The influence of external factors on bacteriophages-review. Folia Microbiol (Praha) 2011;56:191-200.

33. Nobrega FL, Costa AR, Santos JF, Siliakus MF, Van 
Lent JW, Kengen SW, et al. Genetically manipulated phages with improved $\mathrm{pH}$ resistance for oral administration in veterinary medicine. Sci Rep 2016;6:39235.

34. Rahmat Ullah S, Andleeb S, Raza T, Jamal M, Mehmood K. Effectiveness of a lytic phage SRG1 against vancomycin-resistant Enterococcus faecalis in compost and soil. Biomed Res Int 2017;2017:9351017.

35. Marcó MB, Reinheimer JA, Quiberoni A. Phage adsorption to Lactobacillus plantarum: influence of physiological and environmental factors. Int J Food Microbiol 2010;138:270-275.

36. Khanorkar SV (2011). Insights in Physiology. $1^{\text {st }}$ ed. Jaypee Brothers Medical Pub. New Delhi.

37. Ma Y, Pacan JC, Wang Q, Xu Y, Huang X, Korenevsky A, et al. Microencapsulation of bacteriophage felix O1 into chitosan-alginate microspheres for oral delivery. Appl Environ Microbiol 2008;74:4799-4805.

38. Ramirez K, Cazarez-Montoya C, Lopez-Moreno HS, Castro-del Campo N. Bacteriophage cocktail for biocontrol of Escherichia coli O157: H7: stability and potential allergenicity study. PLoS One 2018;13(5):e0195023.

39. Zhang W, Mi Z, Yin X, Fan H, An X, Zhang Z, et al.
Characterization of Enterococcus faecalis phage IMEEF1 and its endolysin. PLoS One 2013;8(11):e80435.

40. Szafrański SP, Winkel A, Stiesch M. The use of bacteriophages to biocontrol oral biofilms. J Biotechnol 2017;250:29-44.

41. Limoli DH, Jones CJ, Wozniak DJ. Bacterial extracellular polysaccharides in biofilm formation and function. Microbiol Spectr 2015;3:10.1128/microbiolspec. MB-0011-2014.

42. Azeredo J, Sutherland IW. The use of phages for the removal of infectious biofilms. Curr Pharm Biotechnol 2008;9:261-266.

43. Melo LDR, Ferreira R, Costa AR, Oliveira H, Azeredo J. Efficacy and safety assessment of two enterococci phages in an in vitro biofilm wound model. Sci Rep 2019;9:6643.

44. Abedon ST. Ecology of anti-biofilm agents II: bacteriophage exploitation and biocontrol of biofilm bacteria. Pharmaceuticals (Basel) 2015;8:559-589.

45. Chan BK, Abedon ST. Bacteriophages and their enzymes in biofilm control. Curr Pharm Des 2015;21:8599. 